\title{
Formação de Fases Quasicristalinas No Sistema $\mathrm{Al}_{65} \mathrm{Cu}_{20} \mathrm{Fe}_{15}$
}

\section{Quasicrystalline Phase Formation in the System $\mathrm{Al}_{65} \mathrm{Cu}_{20} \mathrm{Fe}_{15}$}

\author{
Lourdes Cristina Lucena Agostinho Jamshidi ${ }^{1}$; Luciano Nascimento²; Reza Jamshidi \\ Rodbari ${ }^{3}$ Celmy Maria B. de Menezes Barbosa ${ }^{4}$
}

\begin{abstract}
Resumo
Características estruturais, morfológicos e fases existente na liga quasicristalina com composições nominal de $\mathrm{Al}_{65} \mathrm{Cu}_{20} \mathrm{Fe}_{15}$ foram investigados por difração de raios-X (DRX), Microscopia Eletrônica de Varredura (MEV)/ Espectroscopia de Energia Dispersiva (EDS), Calorimetria Exploratória Diferencial (DSC) e Área Superficial Específica (BET). Os resultados mostram que foram observados vários aspectos morfológicos das fases quasicristalinas em função da sua obtenção em fornos elétricos a indução e a arco em condições experimentais em que ambas as fases cristalinas e quasicristalinos coexistem à $850^{\circ} \mathrm{C}$. Quando a perda de alumínio no interior dos grãos é demasiado elevada, a fase icosaédrica transforma em fase $\beta$ cúbica (tipo de $\mathrm{CsCl}$ ). Devido aos eventos térmicos (exotérmico) numa faixa $400-1200^{\circ} \mathrm{C}$ apresentando aumento do número de fase e a área superficial apresentar um ótimo candidato,podendo ser usado como catalisador em reações catalíticas devido a sua estabilidade térmica. Finalmente futuras aplicações atuais e possíveis de quasicristais Al-Cu-Fe são discutidos à luz de suas propriedades.
\end{abstract}

Palavras-chave: Quasicristal. Catalisador. Fases Quasicristalinas. Caracterizações.

\begin{abstract}
Structural, morphological and existing phases in quasicrystalline alloy with nominal compositions A165Cu20Fe15 were investigated by X-ray Diffraction (XRD), Scanning Electron microscopy (SEM)/ Energy Dispersive Spectroscopy (EDS), Differential Scanning Calorimetry (DSC) and Specific Surface Area (BET). The results show that morphology were observed several of quasicrystalline phases in function of obtaining electric induction furnaces and arc under experimental conditions where both crystalline and quasicrystalline phases coexist at $850^{\circ} \mathrm{C}$. When the aluminum loss inside the grains is too high, the icosahedral phase transforms into $\beta$ cubic phase ( $\mathrm{CsCl}$ type). Due to thermal events (exothermic) in a range $400-1200^{\circ} \mathrm{C}$ for an increase of the phase number and surface area presents an optimal candidate may be used as catalyst in catalytic reactions because of its thermal stability. Finally current and future possible applications of quasicrystals $\mathrm{Al}-\mathrm{Cu}-\mathrm{Fe}$ are discussed in the light of its properties.
\end{abstract}

Keywords: Quasicrystal; Catalyst. Quasicrystalline Phases. Characterizations.

1 Doutora em Engenharia Química, Programa de Pós-Graduação em Engenharia Química, Centro de Tecnologia e GeociênciasCTG/UFPE. cristina.ufcg@gmail.com

2 Doutor em Engenharia Química, Programa de Pós-Graduação em Engenharia Química, Centro de Tecnologia e GeociênciasCTG/UFPE. luciano.ufpe@gmail.com

3 Mestre em Ciências de Materiais, Programa de Pós-Graduação em Ciências de Materiais, Centro de Ciências Exatas e da Natureza-CCEN/UFPE. rodbari.ufpe@gmail.com

4 Professora Titular, Programa de Pós-Graduação em Engenharia Química, Centro de Tecnologia e Geociências-CTG/UFPE. celmy.ufpe@gmail.com 


\section{Introdução}

Quasicristais são sólidos com estrutura atômica quasiperiódica de longo alcance e simetrias rotacionais cristalográficas proibidas para cristais com estruturas periodicamente ordenada (SRIVASTAVA et al., 2014). Por causa da sua estrutura, as ligas quasicristalinas possuem propriedades físico-químicas e mecânicas únicas. Estes materiais podem ser fabricados por moagem mecânica de alta energia, por solidificação rápida ou convencional, deposição física de vapor assistida por plasma (PAPVD). Quasicristais têm muitas propriedades interessantes, como alta dureza, baixa condutividade elétrica e térmica, baixa energia de superfície, acompanhado por um baixo coeficiente de atrito, oxidação razoável, forte resistência à corrosão e propriedades ópticas incomuns que não são observadas em outras ligas cristalinas e ótimos armazenadores de hidrogênio e excelentes propriedades catalíticas (BONASSO; PIGEAT, 2003).

Tais propriedades dos materiais quasicristalinos têm sido exploradas para aplicações de revestimento, armazenamento de hidrogênio e de catalisadores para reações catalíticas usando reagentes de metanol (AGOSTINHO et al., 2013). Até agora, uma série de composições de ligas quasicristalinas foram obtidos em vários sistemas binários, ternários e de vários componentes múltiplos. Normalmente, as fases intermetálicas, amorfas e cristalina em sistemas baseados em Al, Co, Ni, Cr, Fe, Mg, Zr, Ti, Zn e $\mathrm{Cu}$ se formam devidos as transições de fases existentes entre estes elementos que se formam em reação de estado sólido. Como a variedade de elementos metálicos de base que formam as fases quasicristalinas é grande, o espectro destes elementos de liga é ainda maior. No entanto, os elementos de liga são muitas vezes tóxicos, não está facilmente disponível ou ainda muito dispendiosa. As ligas com composição Al-Cu-Fe são uma exceção; eles são interessantes devido a sua falta de toxicidade, fácil disponibilidade e custos razoáveis de compra de seus elementos de liga, devido à grande abundância destes elementos na nutreza (YIN et. al., 2008). Portanto, nas duas últimas décadas, ligas quasicristalinas com composições estequiométricas $\mathrm{Al}-\mathrm{Cu}-\mathrm{TM}$ $(\mathrm{TM}=\mathrm{Fe}, \mathrm{Ni}, \mathrm{Cr}, \mathrm{Nb}, \mathrm{Co}, \mathrm{Ni}, \mathrm{Pd}$ e $\mathrm{Mn})$ têm sido intensamente estudada em vários campos científicos devidos as suas físico-químicas e mecânicas (TSAI, 2008). A maioria destas ligas quasicristalinas é metastável que se transformam de forma irreversível em cristais regulares após tratamento térmico ou em forma amorfas com pequenos cristais de forma irregular. Tem sido relatado que a fase de quasicritalina formada na liga $\mathrm{Al}_{65} \mathrm{Cu}_{20} \mathrm{Fe}_{15}$ convencionalmente solidificado é termodinamicamente estável e não sofre transformação de fases bruscas (pode existir pequenas regiões de traços amorfos e intermetálicos) até ao ponto de fusão, a $1135 \mathrm{~K}$ (GOGEBAKANA et al.,2011).

Portanto, a descoberta destas fases quasicristalinas termodinamicamente estáveis em liga $\mathrm{Al}_{65} \mathrm{Cu}_{20} \mathrm{Fe}_{15}$ abriu um novo caminho em suas investigações experimentais. Após a descoberta, o número de sistemas de liga leve, que em quasicristais são formados por solidificação rápida ou convencional, tem vindo a aumentar de forma crescente com várias aplicações; uma delas são materiais catalíticos devidos aos transportes difusivos de sítios ativos existentes na superfície e que em contato com reagentes (metanol, e etc.) formam produtos de interesse comercial como: éter dimetílico, hidrogênio, formaldeído, ácido metanóico e etc. Os métodos de preparação desta ligas ternárias e sua composição, propriedades mecânicas,magnéticas e calorimétricas e químicas dessas ligas quasicristalinas têm sido os principais temas de interesse no campo da ciência de materiais e metalurgia.

As características estruturais e morfológicas, como o comportamento térmico convencionalmente solidificado, foram obtidas por forno elétrico a arco de indução obtida numa temperatura de 
$1200^{\circ} \mathrm{C}$, tendo uma composição de estequiométrica $\mathrm{Al}_{65} \mathrm{Cu}_{20} \mathrm{Fe}_{15}$ que, foram investigados no presente estudo, por difração de raios-X (DRX), Microscopia eletrônica de varredura (MEV) e espectroscopia de energia dispersiva (EDS), Calorímetro Diferencial de Varredura (DSC) e Analisador de Área Superficial BET.

\section{Materiais e Métodos}

Os pós dos elementos Alumínio,Ferro e Cobre, conforme a sua granulometria, tendo uma pureza de 99,9\%, provenientes da Alcoa no Brasil-Alcoa Alumínio e Aldrich Chemical, foram pesados nas proporções adequada à composição $\left(\mathrm{Al}_{65} \mathrm{Cu}_{20} \mathrm{Fe}_{15}\right)$, homeneizados mecanicamente e compactada na forma de disco com diâmetros de $10 \mathrm{~mm}$ e $2 \mathrm{~mm}$ de espessura,em uma prensa hidráulica uniaxial. Inicialmente foi feita uma pré-prensagem sob carga máxima de 5 tonelada durante 3 minutos. A liga precursora foi obtida por fusão direta dos constituintes compactados. A fusão foi realizada em forno a arco sob atmosfera controlada de argônio. O forno elétrico a arco de indução é alimentado por um gerador retificado estático BALMER cuja potência é de 22 KVA. Para a identificação das fases, foi usado um difratômetro Shimadzu XRD 6000, utilizando radiação de $\mathrm{CuK} \alpha$ com comprimento de onda de $\lambda=1,5406 \AA$.

As medidas foram tomadas para uma ampla

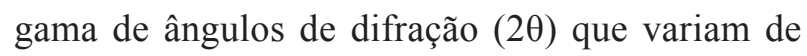
$20^{\circ}$ a $120^{\circ}$ com passo angular de $0,05^{\circ}$ e com tempo de contagem por ponto igual a $2,4 \mathrm{~s}$. A análise em MEV/EDS foi realizada com um SHIMADZU SUPERSCAN SSX-550 com uma tensão de aceleração de 0,5 a $30 \mathrm{kV}$ com uma etapa de $10 \mathrm{~V}$, após a amostra ter sido revestido com uma camada de ouro depositada em vácuo, a fim de melhorar o contraste. As propriedades térmicas das ligas foram analisados por Calorímetro Diferencial de Varredura (DSC) a $10^{\circ} \mathrm{Cmin}^{-1}$ de taxa de aquecimento sob fluxo de $\mathrm{N}_{2}$ e realizado análise porosidade e área Superficial (BET) da marca área de superfície V-Sorb 2800TP.

\section{Resultados e Discussão}

\section{Difração de raios- $X$}

A Figura 1 mostra o difratograma de DRX para amostra da liga quasicristalina $\mathrm{Al}_{65} \mathrm{Cu}_{20} \mathrm{Fe}_{15}$ ilustrado respectivamente a seguir. Os picos de difração identificamas fases; i-fase icosaedral, $\beta$-fase cristalina de composição $\mathrm{Al}_{50-\mathrm{x}}(\mathrm{Cu}, \mathrm{Fe})_{50+\mathrm{x}}$ e esta composição é de cerca de $\mathrm{Al}_{65} \mathrm{Cu}_{20} \mathrm{Fe}_{15}$, em equilíbrio com outras fases cristalinas como $\beta-\mathrm{AlFe}(\mathrm{Cu}), \lambda-\mathrm{Al}_{13} \mathrm{Fe}_{4}$, $\lambda-\mathrm{Al}_{3} \mathrm{Fe}, \quad \theta-\mathrm{Al}_{2} \mathrm{Cu}, \quad \omega-\mathrm{Al}_{7} \mathrm{Cu}_{2} \mathrm{Fe}$ e $\varphi-\mathrm{Al}_{10} \mathrm{Cu}_{10} \mathrm{Fe}_{1}$. Entre essas fases cristalinas, $\omega$ mostra uma grande semelhança com a fase $\beta$ icosaedral. A coordenação dos átomos de Fe é muito semelhante em ambas as estruturas. Além disso, esta fase aparece em três campos de fase. O padrão de difração de raios-X é obtido a partir de composição diferente estudado onde a fase- $\beta$ icosaédrica coexistem com estas fases cristalinas ( $\theta$ é um liquida e soluta) em $850^{\circ} \mathrm{C}$. É interessante ressaltar que a fase $\mathrm{i}$-quasicristalina sempre coexiste com a fase icosaedral e com fase cristalina ( $\beta$ ) (ROSAS et al.,2007).

A solidificação de equilíbrio para esta transformação consiste na formação primária fase $\lambda-\mathrm{Al}_{13} \mathrm{Fe}_{4}$ na fase líquida dando origem a mais da fase $\beta$ por uma reação peritética e, finalmente, a fase-i é formada por uma segundo reação peritética do tipo $\mathrm{L}+\beta+\lambda \rightarrow$ fase-i (LOGRASSO; DELANEY, 1996). É possível que uma fase-i metaestável se forme em baixas temperaturas. No entanto, a sequência de solidificação global do sistema Al-Cu-Fe varia com a velocidade de resfriamento em cada fase formada através de reação do estado sólido, através do tratamento térmico criando fases especiais.

No entanto, um entendimento claro sobre a solidificação peritética, em condição de nãoequilíbrio, ainda não foi atingida. Mesmo assim, em elevada taxa de resfriamento e para subresfriamento que muitas vezes é ignorado a transformação de fase primária $\lambda$ - $\mathrm{Al}_{13} \mathrm{Fe}_{4}$, ocorrendo uma ligeira passivação de camada de óxido intermetálico nesta fase e, portanto a 
inibição da reação peritética para outros estágios avançados de oxidação brusca.

Podemos observar que os picos de difração de fase $\tau$ sobrepõem exatamente com os picos de difração de fase $\beta$ com a (i fase), uma vez que a estrutura de cristal do tipo de $\mathrm{CsCl}(\beta-\mathrm{AlFe}(\mathrm{Cu}))$ cúbica e um parâmetro de rede muito semelhantes $(\mathrm{a}=0,2910 \mathrm{~nm})$.

Quando a perda de alumínio no interior dos grãos é demasiadamente elevada, a fase icosaédrica transforma em fase $\beta$ cúbica (tipo de $\mathrm{CsCl}$ ).

Figura1-Difratograma de DRX da amostra $\mathrm{Al}_{65} \mathrm{Cu}_{20} \mathrm{Fe}_{15}$.

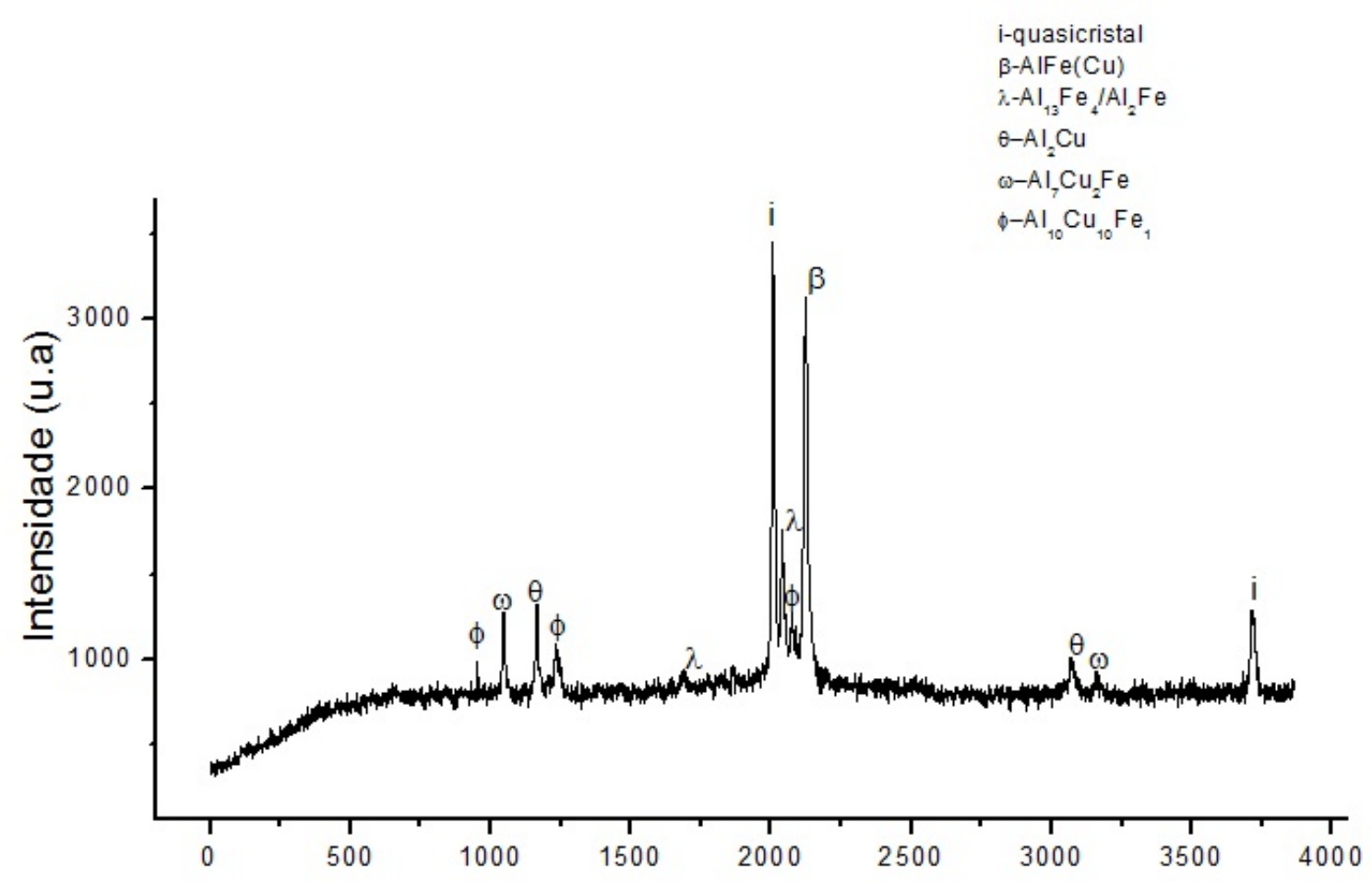

Fonte: Autor

\section{Microscopia eletrônica de varredura e EDS}

Com o objetivo de analisar a dependência composicional microestrutural e espectro de EDS é construído ao plotar valores de intensidade de raios-X contra energia. Os picos correspondem aos diferentes elementos presentes na amostra da liga de quasicristal $\mathrm{Al}-\mathrm{Cu}-\mathrm{Fe}$ obtidas por forno à arco. A análise por microscopia de varredura mostrou diferentes aspectos microestrutural para a liga $\mathrm{Al}_{65} \mathrm{Cu}_{20} \mathrm{Fe}_{15}$, foram observadas quasicristais apreciáveis, de acordo com os resultados de DRX. Isto é provavelmente devido à composição desta liga de fase quasicristalinas. A morfologia desta liga é diversa e algumas partículas exibiam morfologia facetada, com discordâncias e formação de morfologia de "couve-flor" (formato de coração) de acordo com a Figura 2 abaixo, devido fases existentes (intermetálicas associadas com pequenas regiões de óxidos). A nucleação da fase $\beta$ pode ocorrer em sítios, devido a mobilidade dos elétrons de condução de cobre e ferro. As plaquetas crescem 
a partir dessa interface para o núcleo de pequenos cristais em formato de nódulos e de fase icosaedral com um formato de estrutura triangular com discordâncias em seu relevo, que se transformam estruturas com cristais regulares perfeitos onde o teor de ferro e cobre são maiores como é ilustrado na Figura 3. O tamanho de grão deste quasicristal varia de 5 a $10 \mu \mathrm{m}$. Por conseguinte, é realçada uma ligeira alteração na composição (impurezas) que causa uma diferença significativa na evolução macroestrutural durante o processamento metalúrgico. A fim de investigar a alteração estrutural que ocorre durante o tratamento térmico às vezes é indicado e desejado a formação de outros tipos de fases na liga quasicristalinas $\mathrm{Al}-\mathrm{Cu}-\mathrm{Fe}$ para aumentar a formação de fase icosaedral e abri mais os sítios, as amostras podem sofrer um tratamento térmico de $3 \mathrm{~h} \mathrm{a} 850^{\circ} \mathrm{C}$ e, em seguida, resfriada rapidamente para congelar a microestrutura para análises de DRX subsequente e análise do MEV,que é o indicado nestes estudos.

Análise de composição realizada com uma EDS conforme ilustrado na Figura 4 mostra que as regiões foram compostas, principalmente, de $\mathrm{Cu}$, $\mathrm{Fe}, \mathrm{O}$, e uma pequena quantidade de $\mathrm{Al}$, que pode se complexar com o $\mathrm{Cu}, \mathrm{Al}_{2} \mathrm{O}_{3}, \mathrm{AlFe}_{3}, \mathrm{Al}_{3} \mathrm{Cu}, \mathrm{Cu}_{2} \mathrm{O}$ e $\mathrm{Fe}_{3} \mathrm{O}_{4}$ ou $\mathrm{CuFeO}_{2}$ pois as fases presentes na transformação é devido as fortes presenças de elétrons de condução do $\mathrm{Fe}$ e $\mathrm{Cu}$ que são protegidos pela fina camada de óxido de alumínio que possibilita a reação peritética entre a as fases de $\gamma-\mathrm{Al}_{13} \mathrm{Fe}_{4}$ líquido e para formar a fase densa altamente $\omega-\mathrm{Al}_{7} \mathrm{Cu}_{2} \mathrm{Fe}_{1}$. Os nódulos representam gotículas de fase $\beta$ formado principalmente por tensão superficial de líquido (solução saturada provocada por reação no estado sólido) dentro uma bolha ou retração oca (cavidade) solidificada na sua forma líquida inicial. Os quasicristais de forma facetadas de fase são observados nas proximidades da única fase e forma puramente cristalina na fase $\beta$ (SUROWIEC; BOGDANOWICZ; SOZANSKA, 2014). Além disto, o alumínio é mais predominante do que os demais elementos (Cobre e Ferro) em proporções exatas, pois a existência de $\gamma-\mathrm{Al}_{2} \mathrm{O}_{3}$ favorecer a formação de espinélio sobre do cobre e do ferro oxidado formando $\mathrm{CuO}$ e $\mathrm{FeO}$. No entanto, a possibilidade de formação de outros óxidos espinélio como $\mathrm{CuFe}_{\mathrm{x}} \mathrm{Al}_{2-\mathrm{x}} \mathrm{O}_{4}$, são essenciais a catálise de superfície e os compósitos de $\mathrm{CuAl}_{2} \mathrm{O}_{4}$ e $\mathrm{Fe}_{3} \mathrm{O}_{4}$ são complexos que forma um filme fino,que a passiva estrutura da $\mathrm{Al}_{65} \mathrm{Cu}_{20} \mathrm{Fe}_{15}$.

Figura 2- Morfologia de "couve-flor (formato de coração)" da liga quasicristalina $\mathrm{Al}_{65} \mathrm{Cu}_{20} \mathrm{Fe}_{15}$.

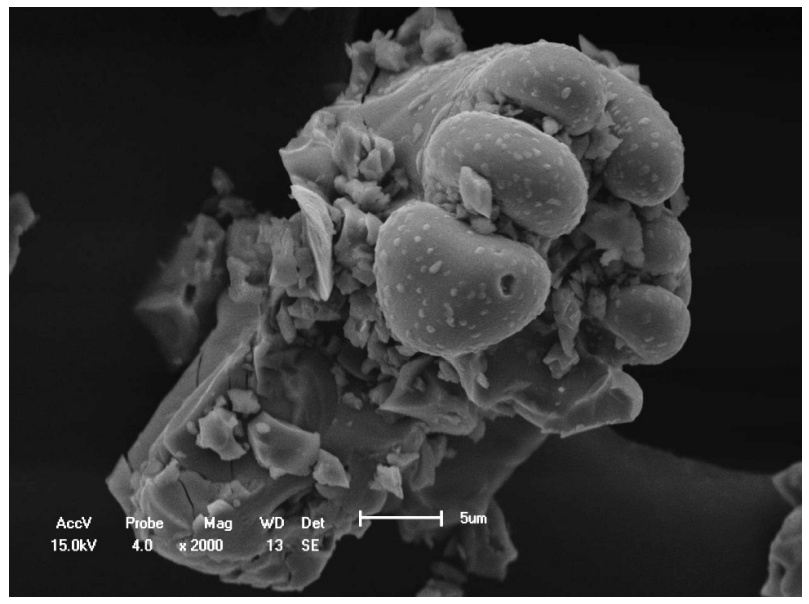

Fonte: Autor

Figura 3- Estrutura triangular (fase $\beta$ ) na fase icosaedral no quasicristal $\mathrm{Al}_{65} \mathrm{Cu}_{20} \mathrm{Fe}_{15}$.

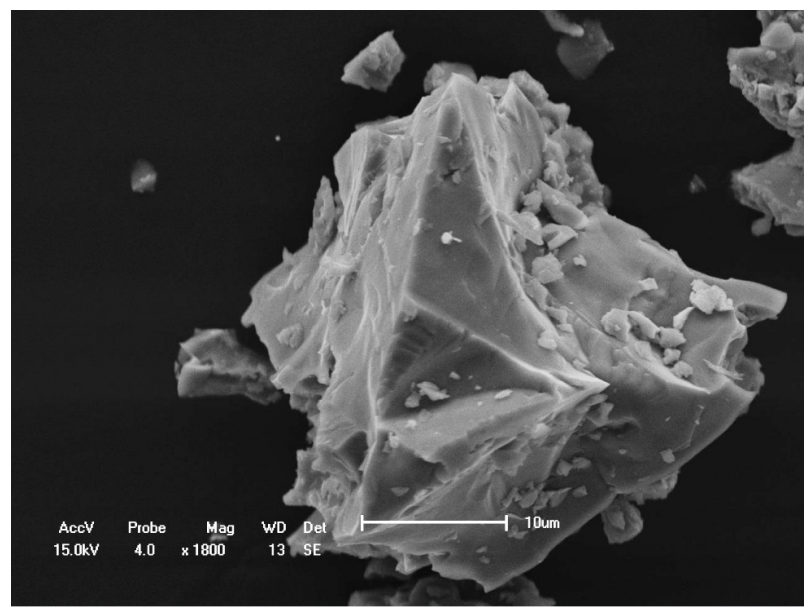

Fonte: Autor 


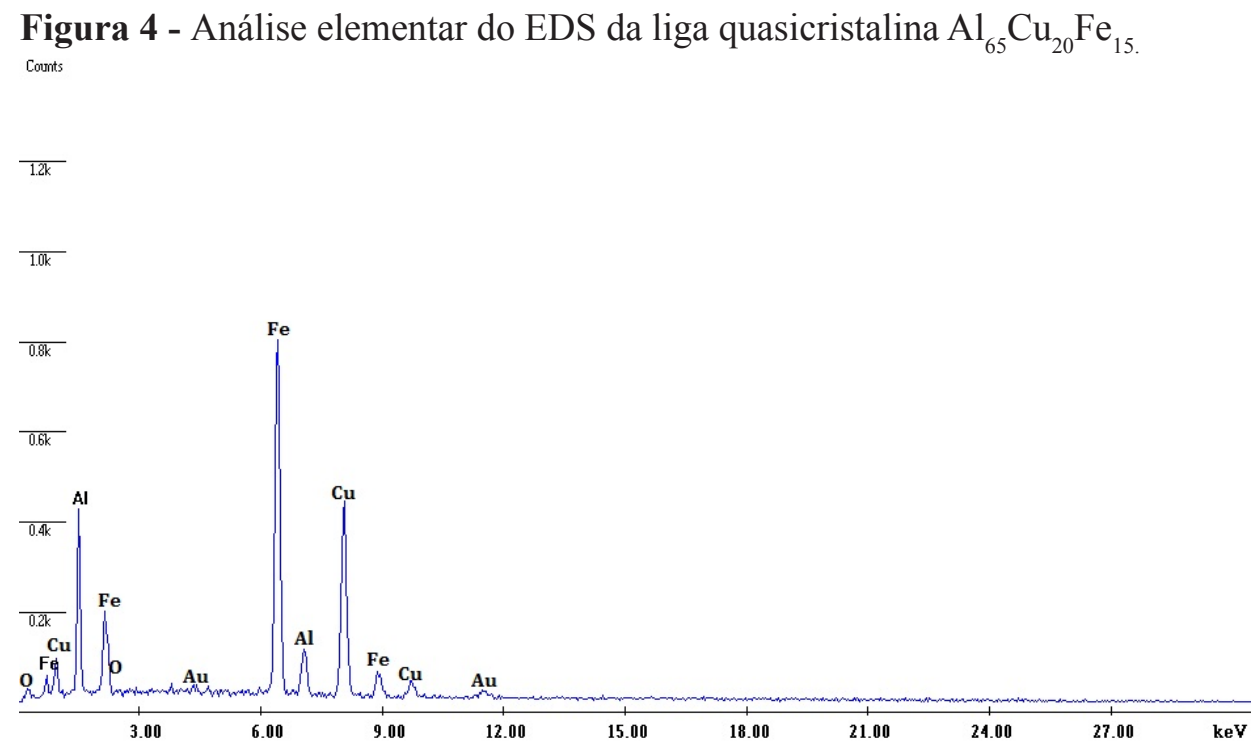

Fonte: Autor

\section{Análise de B.E.T}

A área superficial específica das partículas foi determinada por medição da área superficial B.E.T. A área da superfície de cobre e de dispersão foi determinada por medições $\mathrm{H}_{2}$-TPR de $\mathrm{Cu}$ oxidados por $\mathrm{N}_{2} \mathrm{O}$ a uma taxa de aquecimento $2^{\circ} \mathrm{C} \mathrm{min}^{-1}$ num fluxo de $30 \mathrm{ml} \mathrm{min}^{-1}$ de $5 \%$ de $\mathrm{H}_{2} / \mathrm{Ar}$.

A composição, temperatura de fusão e a área de superfícial BET $\left(\mathrm{S}_{\mathrm{BET}}\right)$ de diferentes amostras de Al$\mathrm{Cu}-\mathrm{Fe}$ estão listadas na Tabela 1 abaixo. A liga foi transformada em pó e peneirada por uma peneira granulométrica de 100 mechas. A área superficial específica das duas ligas observadas $\mathrm{Al}_{62,2} \mathrm{Cu}_{25,3} \mathrm{Fe}_{12,5}$ $\left(31,5 \mathrm{~m}^{2} / \mathrm{g}\right)$ e $\quad \mathrm{Al}_{65} \mathrm{Cu}_{20} \mathrm{Fe}_{15} \quad\left(29,99 \mathrm{~m}^{2} / \mathrm{g}\right)$ tem característica de um ótimo catalisador pois suas áreas superficiais são ótimas para processos catalíticos, pois pode apresentar uma atividade e seletividade catalítica em várias reações (AGOSTINHO, 2009). Tanto uma quanto a outra pode passar por um tratamento térmico que aumenta o número de fases e as camadas espessas de óxido em sua superfície, que mostra excelentes resultados em nível catalítico devido o teor de elementos presentes nas suas composições que oxida e se complexa com o ferro e o cobre formando intermetálicos que aumentando o número de sítios catalíticos.
Tabela 1 - Composição, temp. de fusão (K) e área superficial BET para Ligas quasicristalina $\mathrm{Al}-\mathrm{Cu}-\mathrm{Fe}$.

\begin{tabular}{c||c||c}
$\begin{array}{c}\text { Composição de } \\
\text { Liga }\end{array}$ & $\begin{array}{c}\text { Temp. de } \\
\text { Fusão (K) }\end{array}$ & $\mathbf{S}_{\text {BET }}\left(\mathbf{m}^{2} \mathbf{g}^{-1}\right)$ \\
$\mathrm{Al}_{63} \mathrm{Cu}_{25} \mathrm{Fe}_{12}$ & 973 & 28,98 \\
$\mathrm{Al}_{62,2} \mathrm{Cu}_{25,3} \mathrm{Fe}_{12,5}$ & 1073 & 31,5 \\
$\mathrm{Al}_{65} \mathrm{Cu}_{20} \mathrm{Fe}_{15}$ & 988 & 29,99
\end{tabular}

Fonte: Autor

\section{Análise térmica DSC}

A Figura 5 ilustra o comportamento térmico durante o aquecimento não-isotérmico foi estudado por Calorímetro Diferencial de Varredura (DSC) realizada para uma taxa de aquecimento de $10^{\circ} \mathrm{Cmim}^{-1}$, para o pó da liga quasicristalina $\mathrm{Al}_{65} \mathrm{Cu}_{20} \mathrm{Fe}_{15}$ durante o aquecimento $400-1200^{\circ} \mathrm{C}$, ocorre eventos exotérmicos que são definidos claramente e distinguidos numa faixa de baixa temperatura de $500^{\circ} \mathrm{C}$, presumivelmente relacionada com a formação de fases $\varphi-\mathrm{AlFe}_{3} \mathrm{e} \omega-\mathrm{Al}_{7} \mathrm{Cu}_{2} \mathrm{Fe}$,que representa um evento de fusão ou dissolução das fases (amorfa e intermetálicas) corresponde à fusão das fases ricas em cobre e ferro. 
A sequência mais complexa de eventos térmicos é observada acima de $500^{\circ} \mathrm{C}$. O aumento da taxa de aquecimento provoca uma mudança moderada $\left(\approx 20^{\circ} \mathrm{C}\right)$ das temperaturas de transição de fase no sentido de valores mais elevados, caso contrário, a sequência de eventos térmicos não se altera. A fase $\omega-\mathrm{Al}_{7} \mathrm{Cu}_{2} \mathrm{Fe}$ é o principal precursor da fase $\varphi$-(i$\mathrm{Al}_{65} \mathrm{Cu}_{20} \mathrm{Fe}_{15}$ ) (SVIRIDOVA et al.,2014).

Conforme a homogeneização da composição da liga é gradualmente concluída, $\omega-\mathrm{Al}_{7} \mathrm{Cu}_{2} \mathrm{Fe}$ se torna o principal constituinte de fase. A entalpia do primeiro pico mínimo é menor do que os dos outros dois picos, o que indica que a maior concentração de fases do tipo $\omega-\mathrm{Al}_{7} \mathrm{Cu}_{2} \mathrm{Fe}$ e $\varphi-\left(\mathrm{i}-\mathrm{Al}_{65} \mathrm{Cu}_{20} \mathrm{Fe}_{15}\right)$.

Este resultado salienta ainda a necessidade de uma análise detalhada da evolução microestrutural e da formação e estabilidade de fases constituintes durante os tratamentos não isotérmicas de calor, pois isto possibilita a camada rica de óxidos que é essencial para fins catalíticos.

Figura 5-Curva de DSC do quasicristal $\mathrm{Al}_{65} \mathrm{Cu}_{20} \mathrm{Fe}_{15}$.

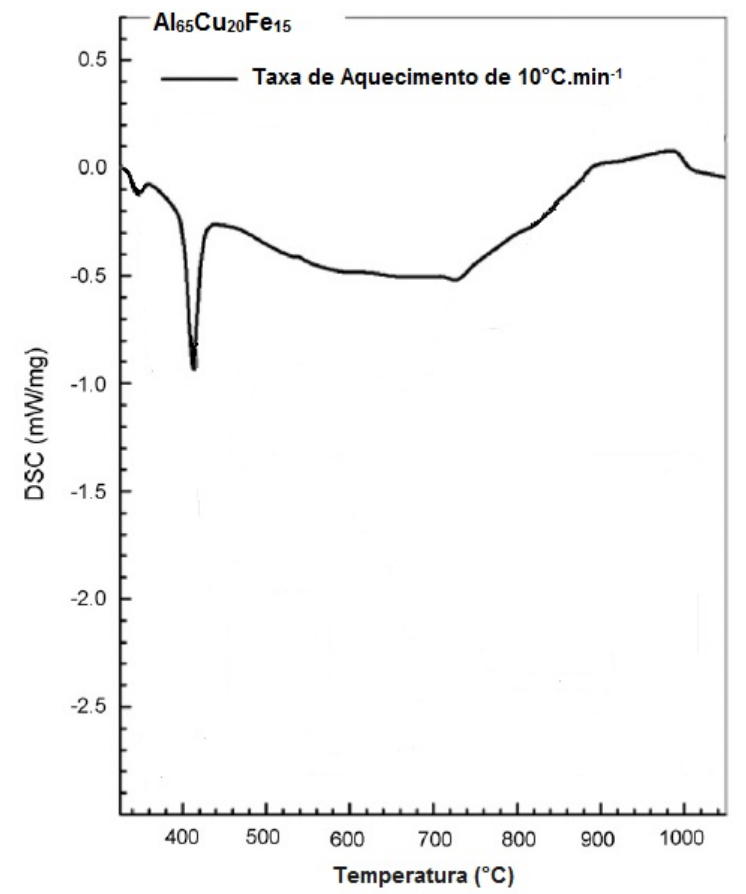

Fonte: Autor

\section{Conclusões}

As principais conclusões da pesquisa são as seguintes:

- No presente estudo, a formação da fase icosaédrica quasicristalinas convencionalmente solidificou ( forno elétrico de indução à arco) em ligas $\mathrm{Al}-\mathrm{Cu}-$ Fe tem sido investigada;

- A microestrutura da liga $\mathrm{Al}_{65} \mathrm{Cu}_{20} \mathrm{Fe}_{15}$ obtida por forno elétrico de indução a arco e solidificada consiste de uma mistura de fase i-quasicristalinas, $\beta, \theta, \omega$ e $\gamma$ em reação de estado sólido;

- É interessante ressaltar que a fase i-quasicristalina sempre coexiste com a fase icosaedral e com a fase cristalina $(\beta)$;

- A fase $\lambda-\mathrm{Al}_{13} \mathrm{Fe}_{4}$ na fase líquida dando origem a mais da fase $\beta$ por uma reação peritética e, formando a fase-i que é formada por uma segunda reação peritética do tipo $\mathrm{L}+\beta+\lambda \rightarrow$ fase- $\mathrm{i}$;

- A perda de alumínio no interior dos grãos é demasiadamente elevada, a fase icosaédrica transforma em fase $\beta$ cúbica (tipo de $\mathrm{CsCl}$ );

- A existência de $\gamma-\mathrm{Al}_{2} \mathrm{O}_{3}$ favorecer a formação de espinélio sobre do cobre e do ferro oxidado formando $\mathrm{CuO}$ e $\mathrm{FeO}$;

- A área superficial específica das duas ligas observadas $\mathrm{Al}_{62,2} \mathrm{Cu}_{25,3} \mathrm{Fe}_{12,5} \quad\left(31,5 \mathrm{~m}^{2} / \mathrm{g}\right) \quad \mathrm{e}$ $\mathrm{Al}_{65} \mathrm{Cu}_{20} \mathrm{Fe}_{15}\left(29,99 \mathrm{~m}^{2} / \mathrm{g}\right)$ tem característica de um ótimo catalisador em reações catalíticas;

- A liga quasicristalina $\mathrm{Al}_{65} \mathrm{Cu}_{20} \mathrm{Fe}_{15}$ durante o aquecimento $400-1200^{\circ} \mathrm{C}$, ocorre eventos exotérmicos que são definidos claramente e distinguidos numa faixa de baixa temperatura de $500^{\circ} \mathrm{C}$, presumivelmente relacionada com a formação de fases $\varphi$ - $\mathrm{AlFe}_{3}$ e $\omega-\mathrm{Al}_{7} \mathrm{Cu}_{2} \mathrm{Fe}$,que representa um evento de fusão ou dissolução das fases (amorfa e intermetálicas). 


\section{Agradecimentos}

Os autores agradecem ao PRH 28/MCT/ ANP pelo o apoio financeiro deste trabalho e ao Laboratório Grupo de Magnetismo e Materiais Magnéticos-MMM do Departamento de Física do Centro de Ciências Exatas e da Natureza da UFPE, Central Analítica do Departamento de Química Fundamental do Centro de Ciências Exatas e da Natureza da UFPE, Alcoa no Brasil-Alcoa Alumínio e ao CETENE.

\section{Referências}

AGOSTINHO, L. C. L.; BARBOSA, C.M.B.M.; NASCIMENTO, L.; RODBARI,J.R. Catalytic Dehydration of Methanol to Dimethyl Ether (DME) Using The $\mathrm{Al}_{62,2} \mathrm{Cu}_{25,3} \mathrm{Fe}_{12,5}$ Quasicrystalline Alloy. Journal Chem. Eng. Process. Technol. 4,pp.28,2013 .

AGOSTINHO, L.C.L. Estudo da Aplicabilidade dos Quasicristais Al-Cu-Fe em Reações Catalíticas na Oxidação do Metanol,Dissertação (Mestrado em Ciências de Materiais),Universidade Federal da Paraíba,João Pessoa-Paraíba,2009.

BONASSO, N.; PIGEAT, P. Preparation of Al$\mathrm{Cu}-\mathrm{Fe}$ ultra-thin quasicrystalline films without protective coatings by MBE: influence of processing. Materials Science and Engineering A 349, pp.224-229, 2003.

GOGEBAKANA,M.; AVAR,B.; TARAKCI,M. Microstructures and mechanical properties of conventionally solidified $\mathrm{Al}_{63} \mathrm{Cu}_{25} \mathrm{Fe}_{12}$ alloy. Journal of Alloys and Compounds 509 S, pp.316319, 2011.

LOGRASSO,T. A.; DELANEY,D. W. Preparation of large single grains of the quasicrystalline icosahedral Al-Cu-Fe $\psi$ phase. Journal of Materials Research 11,pp. 2125- 2127,1996.

ROSAS, G.; REYES-GASGA, J.; PÉREZ, R. Morphological characteristics of the rapidly and conventionally solidified alloys of the AlCuFe system. Materials Characterization 58, pp.765770, 2007.
SRIVASTAVA,V.C.;HUTTUNEN-S AAR IVIRTA, E.; C UI, C.; UHLENWIN KEL, V.; SCHULZ,A.;MUKHOPADHYAY,N.K. Bulk synthesis by spray forming of $\mathrm{Al}-\mathrm{Cu}-\mathrm{Fe}$ and $\mathrm{Al}-$ $\mathrm{Cu}-\mathrm{Fe}-\mathrm{Sn}$ alloys containing a quasicrystalline phase. Journal of Alloys and Compounds 597, pp.258-268, 2014.

SUROWIEC, M.; BOGDANOWICZ, W.; SOZANSKA, M. Evolution of the Phase $\beta$ Flux Dissolution during Quasicrystal Formation. Acta Physica Polonica A 126, pp.594-595, 2014.

SVIRIDOVA, T.A. SHEVCHUKOV, A.P. SHELEKHOV, E.V.; DIAKONOV, D.L.; GORSHENKOV, M.V. KALOSHKIN, S.D. TCHERDYNTSE, V.V. LI, Z.; FAN,G. Nano quasicrystalline Phase in Mechanically Alloyed and Heat-Treated $\quad \mathrm{Al}_{73} \mathrm{Cu}_{11} \mathrm{Cr}_{16}$. Acta Physica Polonica A 126, pp.599-602, 2014.

TSAI,A. P.Icosahedral clusters, icosaheral order and stability of quasicrystals - a view of metallurgy. Sci. Technol. Adv. Mater.9, pp.1-21, 2008.

YIN,S.; LI,C.; BLANB,Q.; LUA,M. Effect of composition on the formability of quasicrystalline phase in mechanically alloyed $\mathrm{Al}-\mathrm{Cu}-\mathrm{Fe}$ powders. Materials Science and Engineering A 496, pp. 362$365,2008$.

Recebido em 27 Abril, 2015 - Received on April 27, 2015 Aceito em 15 Junho, 2015 - Accepted on June 15, 2015 